\title{
Development of transparent ceramic as scintillators
}

\author{
Takayuki Yanagida, and Akira Yoshikawa \\ IMRAM, Tohoku University \\ 2-1-1, Katahira, Aoba-ku, Sendai, Japan \\ E-mail: t_yanagi@tagen.tohoku.ac.jp
}

\section{Kei Kamada}

Material reserch laboratoly, Furukawa, Co. Ltd., 1-25-13, Kannondai, Tukuba, Ibaragi, 305-0856, Japan

E-mail: pinna@mail.tagen.tohoku.ac.jp

\begin{abstract}
Scintillators coupled with photon-detectors have played a major role as radiation detectors. Generally, the term "scintillator" means a single crystalline made. Today, our group try to develop transparent ceramic scintillators, as well as single crystalline scintillators. We investigate optical properties (emission and absorption spectra) and gamma-ray responses (light yield, energy resolution, and decay time) of Ce:YAG, (Ce, Nd):YAG, and Ce:GYAG ceramics, coupled with PMT and APD. For Ce:YAG, energy resolution of $\sim 6 \%$ at $662 \mathrm{keV}$ is achieved when coupled with APD, and this value is better than PMT coupling. In optical properties of codoped material, there are no unexpected middle bands. The stopping power of Ce:GYAG is improved about five times large as that of Ce:YAG in several hundred keV.
\end{abstract}

International workshop on new photon-detectors PD07

Kobe University, Kobe, Japan

27-29 June, 2007 


\section{Introduction}

Inorganic scintillators which are single crystalline, have played a major role as gamma-ray detectors. Recently, ceramic scintillators were developed and start to be used as gamma-ray detectors, instead of single crystalline made ones. Ceramics have some advantages compared with single crystals. They are mechanically strong, easy to obtain large size uniformly, yielding low cost. New chemical compositions, which are impossible in single crystal, are capable. However, a weak point is that to obtain a transparent sample is very difficult.

In mid 1990's, translucent ceramics Ce:YAG, which showed 60\% transparency, were developed [1]. Then in few years ago, we succeeded to develop transparent ceramic Ce:YAG, which showed $\sim 85 \%$ transparency nearly reaching to theoretical limit [2]. Because ceramics are highly uniform, we developed co-doped material, (Ce, Nd):YAG scintillator, and evaluated its radiation and optical properties[3]. Additionally, we succeeded to develop new material, Ce:GYAG which is impossible to be made by crystal growth [4]. In this time, we concentrate on Ce:YAG and Ce:GYAG ceramics coupled with avalanche photodiode (APD), and measure these gamma-ray responses. These samples are shown in figure 1.

\section{Experiment}

Ceramic scintillators are mounted on a light sensitive window of APD (S8664-55, Hamamatsu) with silicone grease. The reason why we use APD is that the emission wavelength of these ceramics are long (500-550 nm). High voltages are supplied to them by CP6621, and radio isotopes are irradiated. An avalanche gain is controlled at 20 times, because we have already investigated that the best energy resolution is achieved around this gain. The signal fed into preamplifier $(\mathrm{CP} 580 \mathrm{H})$, and multiplied at shaping amplifier (CP4417). Finally, we obtain a gamma-ray spectrum by accumulating at MCA (Amptec 8000A). An experimental temperature is controlled at -20 degrees Celcius within 1 degree by a heat bath, because generally APD shows the best performance at low temperature, due to low noise.

\section{Results}

Figure 2 shows gamma-ray spectra of Ce:YAG and Ce:GYAG, both compared with PMT, respectively. Because of high quantum efficiency of APD at longer wavelength, energy resolution at $662 \mathrm{keV}$ is improved in both figures. Compared with direct detection of ${ }^{55} \mathrm{Fe}$ by $\mathrm{Si}$, total light yield of Ce:YAG reaches to $~ 20000 \mathrm{ph} / \mathrm{MeV}$, and Ce:GYAG to $\sim 9000 \mathrm{ph} / \mathrm{MeV}$, respectively. In these figures, peak intensities are normalized to compare energy resolution, but we evaluated detected events number of photo absorption by a single Gaussian fitting. Compared with areas of Gaussian, the stopping power at $662 \mathrm{keV}$ photo-peak achieved $\sim 5$ times as large as that of Ce:GYAG, because of contribution from Gd. This result is also consistent with EGS4 based simulation. The reason why Ce:GYAG shows a low light yield is attributed to its low transparency, $\sim 20 \%$ at emission wavelength. In the development of ceramic Ce:YAG, we could improve the transparency from $60 \%$ to $85 \%$, which is the 
theoretical limit, and we will try to improve the transparency of Ce:GYAG. Decay times are 80 nsec for Ce:YAG, and 90 nsec for Ce:GYAG, respectively. The temperature dependence, and other properties will be represented somewhere.

\section{4. $\quad$ Future prospects}

We succeeded to develop transparent ceramic Ce:YAG, and translucent Ce:GYAG. In future, we are going to improve transparence of Ce:GYAG, by reducing a scattering at grain boundaries. Additionally, we plan to try other chemical compositions, such as Lu or Hf host, taking into account high radiation sensitivity.
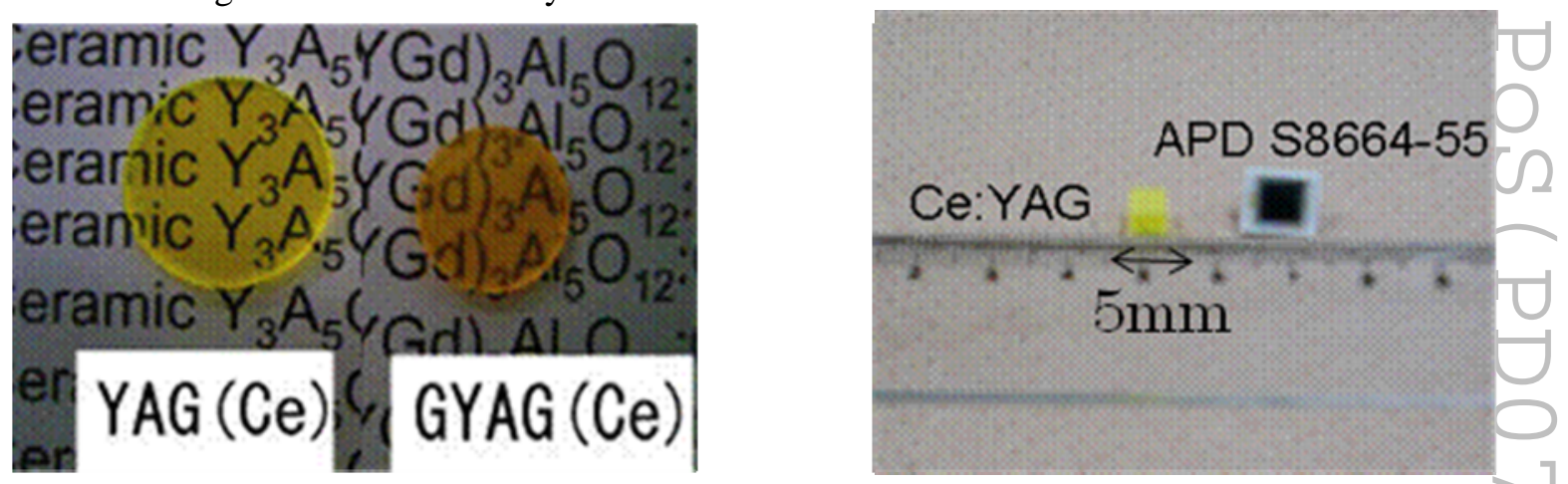

Figure 1. Ceramic Ce:YAG and Ce:GYAG (left), and ceramic Ce:YAG and APD S866455 (right).
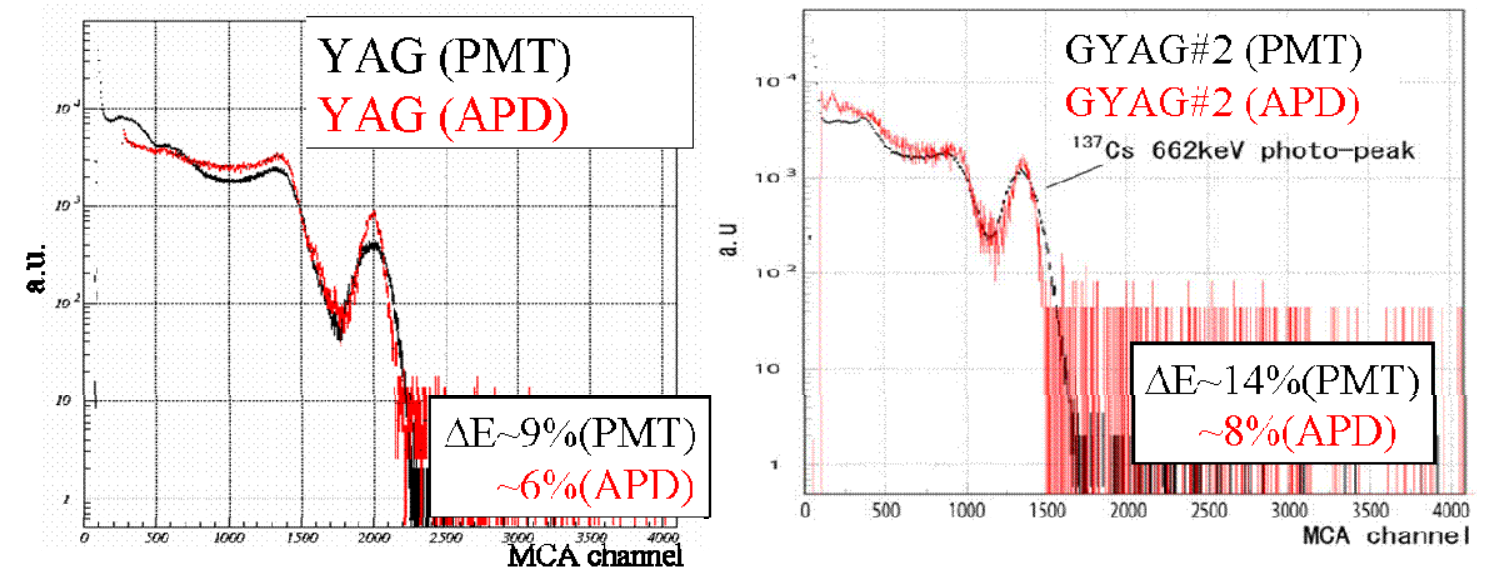

Figure 2. ${ }^{137}$ Cs spectra of Ce:YAG (left), and Ce:GYAG (right), respectively. In both panels, PMT results are superposed to compare with APD results.

\section{References}

[1] E. Zych, et al., Jounal of Luminescence, 75, 193, (1997)

[2] T. Yanagida, et.al., IEEE Trans. Nucl. Sci., 52, 1836 (2004)

[3] T. Yanagida et al., Radiation Detectors and Their Uses, 19, 251 (2006)

[4] T. Yanagida et. al., NIM-A, 579, 23 (2007) 